\title{
Mesenchymal stromal cells coated with anti-ACE2 antibodies might improve efficacy against COVID-19
}

\author{
Nourhan Saied Bakry ${ }^{1}$ (D) Mai Abdelgawad ${ }^{1}$ (D) $\cdot$ Ahmed Abdel-Latif $^{2,3}$ (D) $\cdot$ Ahmed Lotfy $^{1}$ (i)
}

Received: 28 July 2021 / Accepted: 21 September 2021 / Published online: 30 September 2021

(c) Japan Human Cell Society 2021

\section{Introduction}

The life-threatening pandemic COVID-19, emerged in late 2019 , caused by SARS-CoV-2 virus that has been considered a grim reaper with around 5 million death cases worldwide as reported by the WHO. COVID-19 may lead to a cytokine storm that causes a multi-organ damage including acute respiratory distress syndrome (ARDS) and eventually death. SARS-CoV-2 is a positive-sense single-stranded RNA virus with prominent structural and nonstructural proteins. Spike protein (S-protein) is the most crucial structural protein as it participates dramatically in the process of viral invasion into host cells. The viral entry, mediated by the $\mathrm{S}$-protein, is mediated by the cell surface receptor known as angiotensin-converting enzyme2 (ACE2) in human cells [1, 2]. Mesenchymal stromal cell (MSCs)-based therapy is an emerging therapeutic technique for the treatment of a multitude of inflammatory diseases, including ARDS, due to their immunomodulatory and regenerative effects at the site of inflammation [2]. Here, we hypothesize a way to combat SARS-CoV-2 infection by enhancing the homing capacity of MSCs to ACE2-expressing cells and simultaneously hindering SARS-Cov-2 entry into ACE2-expressing cells. Thus, we converge two approaches to augment the therapeutic

Nourhan Saied Bakry, Mai Abdelgawad have contributed equally.

Ahmed Abdel-Latif

abdel-latif@uky.edu

$\triangle$ Ahmed Lotfy

ahmed.lotfy@psas.bsu.edu.eg; lotfy_bio@hotmail.com

1 Biotechnology and Life Sciences Department, Faculty of Postgraduate Studies for Advanced Sciences (PSAS), Beni-Suef University, Beni-Suef 62511, Egypt

2 Gill Heart Institute and Division of Cardiovascular Medicine, University of Kentucky and the Lexington VA Medical Center, Lexington, KY, USA

3 College of Medicine, University of Kentucky, Lexington, KY 40506-0046, USA effects of MSCs solely targeting ACE2-expressing cells coupled with altering the main site for SARS-CoV-2 entry.

\section{Anti-ACE2-coated MSCs may confer a better way for the treatment of COVID-19}

The immune response to SARS-CoV-2 infection encompasses production of massive amounts of chemokines and cytokines, thereby leading a strong cytokine storm; the most remarkable cause of increasing COVID-19 morbidity and mortality rates. These cytokines include: IL-2, IL-6, IL-7, GSCF, IP10, MCP1, MIP1A, and TNF $\alpha$, that upon their release edematous changes occur that lead to impairment in air exchange, ARDS, cardiac injury, organ failure and eventually death. Therefore, antivirals alone is not enough for COVID-19 treatment; instead, a combination of anti-inflammatory drugs, immunomodulators that tackle a multitude of inflammatory mediators (IMs), rather than conventional immunotherapy that target one or two IMs, is required to ameliorate the immune complications of the cytokine storm.

MSCs have great a potential to migrate to injured tissue and localize to the inflamed affected area and exert their regenerative and immunomodulatory effects, thereby they were harnessed as a potential therapy against SARS-CoV-2 infection [2]. For instance, a number of clinical studies was performed to assess toxicity and efficacy of MSCs in COVID-19 [3]. In a study by Lei Shu et al., an improvement in the clinical symptoms in addition to radiological and laboratory values in patients underwent MSCs transplantation in comparison to control group, with no adverse effects, was reported [4]. However, cell retention is a major obstacle for MSCs, and the homing process is inadequate. Furthermore, the concentration of MSCs remaining in the affected area decreases due to the rapid fluid flow accompanying MSCs injection, which causes the cells to be swept away. Consequently, a decline in the ability of MSCs to modulate the inflamed area is observed; for instance in myocardial 


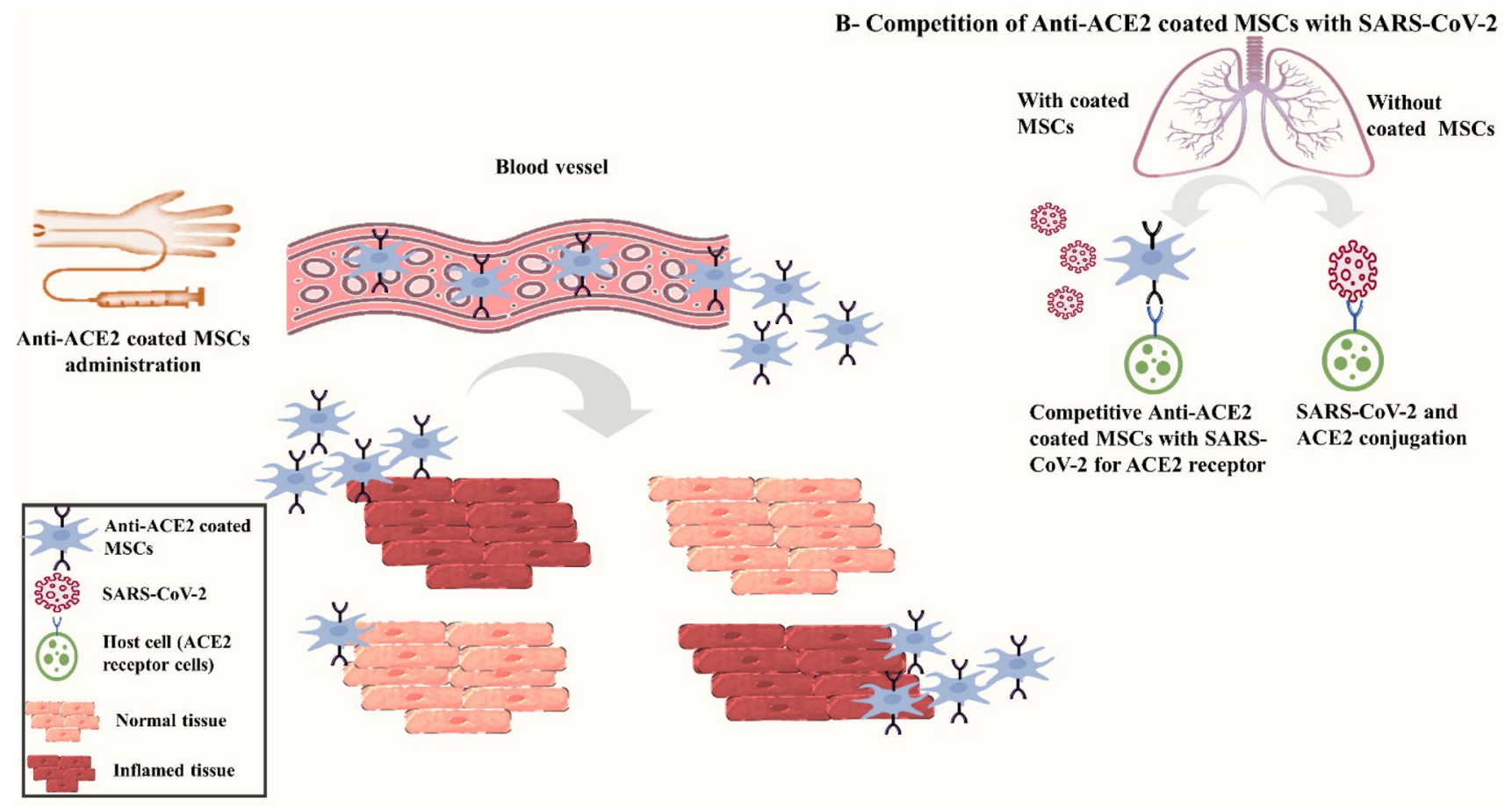

A- Better homing and migration capability of Anti-ACE2 coated MSCs to the inflamed tissue

Fig. 1 Graphical abstract illustrating. A Anti-ACE2-coated MSCs' homing characteristics to diseased and inflamed tissue. MSCs coated with anti-ACE2 migrate through blood vessels to the inflamed tissue to conduct their anti-inflammatory and immunomodulatory functions. Because of the presence of ACE2 receptors, only a small number of anti-ACE2-coated MSCs can reach healthy tissue. Moreover,

infarction $[5,6]$. This, in turn, requires the injection of a high number of cells, which is life-threatening.

To overcome the above-mentioned limitation of MSCs, we suggest the MSCs coating approach that has successful outcomes. Antibody coating plays a significant role in tissue engineering and regenerative medicine. The indirect coating enhances MSCs features. In a trial to enhance MSCs attachment to the decellularized scaffold of a pig's aortic valve, the natural scaffold was coated with antibody against CD90. The result demonstrated better MSCs binding and antibody trapping in the scaffold even under high shear conditions, which reflected the importance of antibody coating in MSCs immobilization and attachment to the engineered scaffold [7]. In another study, MSCs were coated with antibodies on their surface to enable them to adhere to well-defined molecules present on the endothelium of the infarcted heart, known as intercellular cell adhesion molecules-1 (ICAM1), the coated cells displayed threefold better cell retention than the uncoated cells [8]. Consequently, due to the need for effective treatment against COVID-19, we hypothesize that a new potential therapy may limit the infection of SARSCoV-2. We assume that coating MSCs with anti-ACE2 antibodies would be beneficial and may aid in migration and not all MSCs migrate to the inflamed tissue, a few make their way to the healthy one. B Schematic illustration of the competitive effect of anti-ACE2-coated MSCs toward SARS-CoV-2 for ACE2 cells. The coating improves MSC targeting to cells, suggesting their competition with the virus toward the host cells.

homing. It might achieve better cell attachment to the site of infection and compete with SARS-CoV-2 for the same receptor (Fig. 1).

We propose coating MSCs surfaces anti-ACE2 antibodies. Anti-ACE2-coated MSCs will be beneficial for two purposes. First, as discussed previously, MSCs' homing to injured tissue is low. MSCs should home abundantly to injured tissue by coating them with the antigen expressed on the injured tissue, which would direct the antibody-coated MSCs to the specific tissue. Therefore, the therapeutic potential of these licensed MSCs, whether immunomodulatory, anti-inflammatory, or regenerative, will be dedicated primarily to the inflamed tissue (or antigen-expressing tissue). Secondly, binding of those anti-ACE2-coated-MSCs to the extracellular site of ACE2 will preclude the SARSCoV-2 S-protein from binding and entering cells. The antibody should be directed to the metallopeptidase domain (PD) (residues: 19-611) of ACE2, as this is the domain that interacts with the $\mathrm{S}$-protein. In addition, since transmembrane serine protease 2 (TMPRSS2) cleaves the residues (697-716) from ACE2, as a priming step for ACE2 binding to the S-protein, these residues would not become the target or a partial target for the antibody [9]. This would allow the 
anti-ACE2-coated MSCs to target SARS-CoV-2-infected and uninfected ACE2-expressing cells. Subsequently, these anti-ACE2-coated MSCs may be promising as a prophylaxis or treatment for COVID-19. Thus, harnessing anti-ACE2coated MSCs might combat COVID-19 via dual beneficial mechanisms.

Regarding to the main physiological functions mediated by ACE2' enzymatic activity in the body that are controlling blood pressure and cardiovascular homeostasis, maintaining a cardiovascular anti-hypertrophic effect and facilitating efficient vasodilation [10], the application of anti-ACE2-coated MSCs in the setting of COVID-19 may generate undesirable effects due to the inhibition of the enzymatic activity of ACE2. Therefore, there is a risk that ACE2 inhibition would cause vasoconstriction, which could cause an increase in blood pressure and diminished (or even demolished) cardiac protection; therefore, anti-ACE2-coated-MSCs should be used with caution in patients suffering from hypertension and/or cardiovascular comorbidities.

Acknowledgements The authors would like to thank all healthcare workers in Egypt, US, and worldwide for their efforts against this pandemic.

Author contributions The article was written by NSB, MA, and AL. MA and NSB contributed equally to manuscript preparation. AA and AL contributed equally to the critical review of the manuscript. All the authors developed the hypothesis and, read and approved the final form of the article.

Funding Not applicable.

Data availability All data presented in this article are totally available and present in the text.

Code availability Not applicable.

\section{Declarations}

Competing interests No potential conflicts of interest were disclosed.

\section{References}

1. Naqvi AAT, Fatima K, Mohammad T, Fatima U, Singh IK, Singh A, et al. Insights into SARS-CoV-2 genome, structure, evolution, pathogenesis and therapies: Structural genomics approach. Biochim Biophys Acta Mol Basis Dis. 2020;1866(10):165878.

2. Atluri S, Manchikanti L, Hirsch JA. Expanded umbilical cord mesenchymal stem cells (UC-MSCs) as a therapeutic strategy in managing critically ill COVID-19 patients: the case for compassionate use. Pain Phys. 2020;23(2):E71-83 (Epub 2020/03/28).

3. Abdelgawad M, Bakry NS, Farghali AA, Abdel-Latif A, Lotfy A. Mesenchymal stem cell-based therapy and exosomes in COVID-19: current trends and prospects. Stem Cell Res Therapy. 2021;12(1):469 (Epub 2021/08/23).

4. Shu L, Niu C, Li R, Huang T, Wang Y, Huang M, et al. Treatment of severe COVID-19 with human umbilical cord mesenchymal stem cells. Stem Cell Res Therapy. 2020;11(1):361 (Epub 2020/08/20).

5. Barbash IM, Chouraqui P, Baron J, Feinberg MS, Etzion S, Tessone A, et al. Systemic delivery of bone marrow-derived mesenchymal stem cells to the infarcted myocardium: feasibility, cell migration, and body distribution. Circulation. 2003;108(7):863-8 (Epub 2003/08/06).

6. Dow J, Simkhovich BZ, Kedes L, Kloner RA. Washout of transplanted cells from the heart: a potential new hurdle for cell transplantation therapy. Cardiovasc Res. 2005;67(2):301-7 (Epub 2005/05/24).

7. Ye X, Zhao Q, Sun X, Li H. Enhancement of mesenchymal stem cell attachment to decellularized porcine aortic valve scaffold by in vitro coating with antibody against CD90: a preliminary study on antibody-modified tissue-engineered heart valve. Tissue Eng Part A. 2009;15(1):1-11 (Epub 2008/09/02).

8. Wu PJ, Peng H, Li C, Abdel-Latif A, Berron BJ. Adhesive stem cell coatings for enhanced retention in the heart tissue. ACS Appl Bio Mater. 2020;3(5):2930-9 (Epub 2020/11/24).

9. Yan R, Zhang Y, Li Y, Xia L, Guo Y, Zhou Q. Structural basis for the recognition of SARS-CoV-2 by full-length human ACE2. Science. 2020;367(6485):1444-8 (Epub 2020/03/07).

10. Wang W, McKinnie SM, Farhan M, Paul M, McDonald T, McLean B, et al. Angiotensin-converting enzyme 2 metabolizes and partially inactivates Pyr-Apelin-13 and Apelin-17: physiological effects in the cardiovascular system. Hypertension. 2016;68(2):365-77 (Epub 2016/05/25).

Publisher's Note Springer Nature remains neutral with regard to jurisdictional claims in published maps and institutional affiliations.

Ethics approval and consent to participate Not applicable.

Consent for publication Not applicable. 